\title{
PERCURSOS INDIVIDUAIS DE CONSTRUÇÃO DA INTERFONOLOGIA VOCÁLICA PORTUGUÊS BRASILEIRO/INGLÊS-L2
}

\author{
INDIVIDUAL PATHWAYS FOR BUILDING BRAZILIAN \\ PORTUGUESE/ENGLISH-L2 VOWEL INTERPHONOLOGY
}

\author{
Andréa Moniky Moraes de Freitas ${ }^{19}$ \\ Clerton Luiz Felix Barboza ${ }^{20}$
}

\begin{abstract}
RESUMO: Esta pesquisa tem como objetivo geral descrever a emergência da interfonologia vocálica do português brasileiro (PB) e do inglês-L2 na realização de aprendizes brasileiros a partir de uma perspectiva linguística complexa, multirrepresentacional e focada no indivíduo. Buscamos responder à seguinte pergunta problema: de que maneira emerge a construção da interfonologia PB/inglês-L2? Temos por hipótese básica a auto-organização individual no percurso de construção da gramática fonológica do inglês-L2 por aprendizes brasileiros. O estudo defende que a análise da variação individual, normalmente descartada, oferece novas possibilidades de análise linguística, aprofundando o conhecimento do percurso de construção da interfonologia vocálica envolvendo o continuum PB/inglês-L2. O paradigma teórico do estudo envolve a visão de língua(gem) enquanto Sistema Adaptativo Complexo (SAC), bem como os modelos fonológicos multirrepresentacionais, a Fonologia de Uso (FU) e o Modelo de Exemplares (ME). A metodologia envolve um corte transversal e desenho quase-experimental. O universo de pesquisa compreende aprendizes de inglês-L2 de cursos livres de idiomas na cidade de Mossoró-RN. Resultados indicam a relevância do estudo do detalhe fonético de realização das vogais do inglês-L2 por aprendizes brasileiros como um auxiliar no entendimento do percurso individual de construção da interfonologia PB/inglês-L2. Foram observados comportamentos não-lineares, característicos de sistemas complexos à beira do caos, como a emergência de fenômenos de difícil explicação por meio das teorias fonológicas tradicionais, reducionistas e afeitas à descrição de processos e regras fonológicas.
\end{abstract}

PAlAVRAS-ChAVE: Sistemas Adaptativos Complexos. Fonologia de Uso. Modelo de Exemplares. Interfonologia. Auto-organização individual.

\begin{abstract}
This research has as its main objective to describe the emergence of vowel interphonology involving Brazilian Portuguese (BP) and English-L2 as realized by Brazilian learners through a complex, multirepresentational and individual-based perspective. It aims to answer the research-question: how does $\mathrm{BP} /$ English-L2 interphonology emerge? It is hypothesized the construction of individual self-organized pathways by Brazilian learners. This study defends that the analysis of individual variation, normally discarded, offers new possibilities for linguistic analysis, deepening the knowledge associated to the buildup of BP/English-L2 vowel interphonology continuum. The theoretical paradigm is associated to a view of language as a Complex Adaptive System (CAS), alongside with multi-representational phonological models, Usage-Based Phonology (UBP) and the Exemplar Model (EM). As regards methodology, this is a cross-sectional study using a quasi-experimental design. Informants were selected among English-L2 learners in Mossoró-RN-Brazil. Results indicate the relevance of fine phonetic detail associated to English-L2 vowel realization by Brazilian learners as an aid to the understanding of individual pathways for building BP/English-L2 interphonology. Non-linear behavior was observed, characteristic of complex systems at the edge of chaos, with the emergence of phenomena hardly explainable through the use of traditional, reductionist and processes of a rule-driven phonological theory.
\end{abstract}

KEYWORDS: Complex Adaptative Systems. Phonology of Use. Exemplar Model. Interphonology. Individual self-organization.

\footnotetext{
${ }^{19}$ Mestre em Ciências da Linguagem pelo Programa de Pós-Graduação em Ciências da Linguagem - PPCL, da Universidade do Estado do Rio Grande do Norte - UERN. Professora da rede estadual de ensino do estado do Rio Grande do Norte. E-mail: andreammfd@gmail.com

${ }^{20}$ Doutor em Linguística pela Universidade Federal do Ceará - UFC. Docente do Programa de Pós-Graduação em Ciências da Linguagem - PPCL, da Universidade do Estado do Rio Grande do Norte - UERN. E-mail: clertonluiz@gmail.com
} 


\section{Introdução}

Esta pesquisa tem como objetivo geral descrever a emergência da interfonologia vocálica do português brasileiro (PB) e do inglês-L2 na realização de aprendizes brasileiros a partir de uma perspectiva linguística complexa, multirrepresentacional e focada no indivíduo. Buscamos responder à seguinte pergunta problema: de que maneira emerge a construção da interfonologia $\mathrm{PB} /$ inglês-L2? Temos por hipótese básica a auto-organização individual no percurso de construção da gramática fonológica do inglês-L2 por aprendizes brasileiros. Defendemos que a análise da variação individual, normalmente descartada, oferece novas possibilidades de análise linguística, aprofundando o conhecimento do percurso de construção da interfonologia vocálica envolvendo o continuum PB/inglês-L2.

A análise da variação individual é fundamental à língua(gem) enquanto SAC, uma vez que esta emerge a partir do uso individual de cada agente, não sendo associada a um hipotético conhecimento inato do ser humano (DE BOT, 2008; BECKNER et al, 2009; BACCHI, 2015). Adicionalmente, a análise do detalhe fonético é essencial às teorias fonológicas multirrepresentacionais, uma vez que para a FU (BYBEE, 2000, 2002a, 2002b, 2008, 2010) e o ME (PIERREHUMBERT, 1999, 2001, 2002, 2016) a representação mental constitui-se de exemplares mais ou menos prototípicos, cuja existência decorre da percepção e uso de rotinas neuromotoras. Portanto, as teorias linguísticas baseadas no uso não reconhecem dicotomias caras ao pensamento linguístico tradicional, como língua-fala/competênciadesempenho/ fonética-fonologia ${ }^{21}$, propondo uma análise holística dos fenômenos linguísticos.

Nesta pesquisa optamos por analisar o detalhe fonético de realização dos pares vocálicos i:-I, $\mathfrak{x}-\boldsymbol{\varepsilon}$ e u:- $\boldsymbol{\mho}$ do inglês-L2. A aquisição de novas vogais do inglês-L2 é considerada desafiadora para aprendizes brasileiros, uma vez que Flege $(1995,1999)$ aponta que sons de uma L2 mais próximos da L1 são mais desafiadores que sons distintos, uma vez que aprendizes tendem a não percebê-los como diferentes. Pesquisas anteriores relacionadas à produção e percepção de sons vocálicos do inglês-L2 (BAPTISTA, 2000; RAUBER, 2006; BION et al., 2006; BARBOZA, 2008; LIMA JR., 2012; RAMIRES, 2016) apontam que aprendizes brasileiros apresentam dificuldade na realização de alguns dos pares vocálicos apresentados anteriormente. $O$ fato é frequentemente associado à ausência de conhecimento explícito envolvendo a fonologia do inglês-L2 (ZIMMER, 2016).

Este artigo encontra-se organizado em mais três seções, além desta primeira que apresenta sua introdução. A segunda seção foca na discussão de aspectos das teorias norteadoras do artigo. A terceira foca na descrição dos passos metodológicos de coleta e análise de dados. Finalmente, a última seção apresenta as considerações finais do estudo. Passamos à discussão do referencial teórico a seguir.

\section{Paradigmas teóricos}

A teoria dos Sistemas Adaptativos Complexos (SACs) teve início nos estudos sobre sistemas caóticos, cujo objetivo inicial era investigar a variação e não o estado final dos fenômenos da natureza (GLEICK, 1987). Os primórdios da teoria foram propostos por Lorenz (1963), que investigou fenômenos meteorológicos que tinham em comum comportamento caótico; dinâmico; não-linear; imprevisível e sensível às condições iniciais. Tais estudos deram origem ao que Gleick (1987) denominou Teoria do Caos.

\footnotetext{
${ }^{21}$ Nesta pesquisa seguimos os preceitos da Fonologia de Uso e do Modelo de Exemplares, que advogam pela não distinção entre os níveis fonético e fonológico. Portanto, no decorrer do texto optamos pelo uso do negrito para destacar símbolos que carregam ao mesmo tempo informação fonética e fonológica, abolindo o uso de [...] e /.../ para denotar respectivamente transcrições fonéticas ou fonológicas.
} 
O termo Caos refere-se ao período de aparente aleatoriedade, não-linearidade e imprevisibilidade característico dos sistemas complexos. O estudo do Caos influenciou diretamente a visão dos SACs, uma vez que envolve a descrição do comportamento dos agentes responsáveis pela variação nos sistemas. Um SAC é um sistema que envolve múltiplos agentes e elementos, cuja interação acarreta mudanças ao longo do tempo (LARSEN-FREEMAN; CAMERON, 2008).

Entre as características dos SACs, estão a dinamicidade, complexidade e nãolinearidade. A primeira se refere à constante transformação e variação ao longo do tempo. Waldrop (1993) explica que os estudos da complexidade preocupam-se em maior medida com a variação do sistema, e não com a estabilidade. A palavra "complexidade" denota a interação presente no sistema, que é produto do comportamento individual de seus componentes ou agentes. Por sua vez, a não-linearidade sugere a ideia de que efeitos podem ser desproporcionais às causas num sistema complexo.

Os agentes de um SAC são diferentes dos agentes de um sistema simples em termos de número e heterogeneidade. Os agentes de um SAC podem ser, simultaneamente, outros SACs, caracterizando-se como subsistemas de um sistema maior (LEWIN, 1992). De Bot, Lowie e Verspoor (2007) reforçam que os SACs são baseados na visão de que cada sistema é parte de outro sistema, com diversos níveis de complexidade.

Além disso, os SACs apresentam comportamento emergente, são caracterizados como sistemas abertos, sensíveis às condições iniciais e adaptáveis (CAPRA, 1983). A emergência está relacionada ao surgimento de padrões inovadores, resultado da interação entre os componentes ou agentes. Os SACs também são considerados abertos porque interagem com o ambiente através da troca de informações, evoluindo e ganhando complexidade, absorvendo energia do ambiente e da interação entre os agentes (CILIERS, 1998). Eles são sensíveis às condições iniciais, uma vez que variação nessas condições, por menor que seja, dificulta ou mesmo impossibilita predições de comportamento futuro do sistema. A adaptabilidade referese ao constante ajustamento dos SACs às modificações em seu meio ambiente e no próprio sistema. Como esses sistemas são abertos a novos estímulos, com a maior absorção de energia do ambiente, a complexidade do sistema aumenta (HOLLAND, 1995). Ao considerar o percurso de ensino/aprendizagem como um SAC, Kramsch (2012) pontua que dentro desse sistema estão diversos outros subsistemas, como a sala de aula; os alunos; o(s) professor(es); a própria língua. Todos sofrem influência da emergência de comportamentos dos agentes e de elementos do ambiente em que estão inseridos.

Outro conceito relevante na teoria do SAC é o de atrator: um entre vários estados possíveis de um sistema, pelo qual ele é motivado a se estabilizar por um intervalo de tempo indeterminado (COOPER, 1999). Perturbações provenientes do interior ou exterior do sistema podem forçá-lo a mover-se de um atrator para outro, causando variação. A língua está em constante mudança, influenciada por atratores, ou estados de atração, associados a inúmeros elementos ou agentes, linguísticos e não linguísticos. A visão de SAC defendida por LarsenFreeman (1997) retrata esse estado de movimento aparentemente aleatório e imprevisível de forma semelhante ao percurso de aquisição de L2 pelo aprendiz, uma vez não se pode prever em que momento o conhecimento será internalizado pelo falante.

A descrição e análise da variação constituem, portanto, o ponto principal da teoria dos SACs (VAN GEERT; VAN DIJK, 2002). Com isso, ao relacionarmos a teoria dos SACs com o percurso da construção fonológica de aprendizes de inglês-L2, consideramos a variabilidade como propriedade inerente ao conhecimento gramatical. A perspectiva teórica adotada permite ainda incorporar a variabilidade lexical, bem como as particularidades individuais. Seguimos a visão de língua(gem) enquanto SAC devido ao reconhecimento de que parâmetros linguísticos e não-linguísticos atuam na organização da gramática dos aprendizes 
(LARSEN-FREEMAN; CAMERON, 2008). Para De Bot, Lowie e Vespoor (2007), os termos "variação" e "mudança" (linguística) podem ser associados à dinamicidade do uso linguístico.

Tendo em vista as diversas características associadas aos SACs, buscamos neste trabalho analisar a variação individual de aprendizes brasileiros de inglês-L2 decorrente do uso linguístico. Defendemos que a análise dos percursos individuais de construção da gramática fonológica do inglês-L2 por aprendizes brasileiros tem o potencial de aumentar significativamente o entendimento sobre o objeto de estudo. Passamos nos próximos parágrafos à discussão das principais características da Fonologia de Uso (FU) e do Modelo de Exemplares (ME), paradigmas de análise fonológica deste estudo. Também chamadas de teorias fonológicas multirrepresentacionais, os referidos paradigmas coadunam-se com a visão de língua(gem) enquanto SAC e com o foco na variação defendida anteriormente.

A FU enfatiza a importância do uso na aquisição da linguagem ao passo que associa produções fonológicas ao comportamento neuromotor, segundo Bybee (2002a) o comportamento neuromotor se torna mais fluente com a prática. Ao contrário das teorias linguísticas tradicionais, a FU apoia a perspectiva de que a gramática fonológica é uma organização cognitiva resultante das experiências dos falantes. Nessa visão da FU, anteriormente mencionada, enquanto usuários da língua estão em contato com ocorrências (tokens) da língua em uso, eles as categorizam em diferentes níveis de abstrações. $\mathrm{O}$ fenômeno da categorização cria uma vasta rede de associações fonológicas, semânticas e pragmáticas que sobrepõe o que tradicionalmente tem sido designado como léxico e gramática (BYBEE, 2002b). A repetição, a experiência e o tempo de exposição a uma dada língua afetam a maneira como o sistema cognitivo dos falantes categorizam as ocorrências e as estruturas com as quais estão associadas.

Pierrehumbert (2006, p. 13) também advoga que "Modelos baseados no uso acomodam descobertas propondo que representações mentais e padrões do sistema fonológico da Língua-alvo são gradualmente construídos através da experiência com a fala”. Bybee (2000), por sua vez, já propunha explorar o detalhe fonético da L2, pois a forma como o indivíduo armazena os sons à sua estrutura subjacente é proporcional à percepção acústica, e muito embora esse sujeito não consiga articular um som da L2 conforme o input, ele ainda é capaz de fazer distinção entre segmentos semelhantes (BYBEE, 2001).

Bybee e Beckner (2010, p. 829) ratificam a perspectiva da repetição, quando afirmam: “ (...) uma característica geral da cognição é que a repetição de uma atividade tem um efeito cumulativo no comportamento futuro." Os autores afirmam que a repetição faz desenvolver o conhecimento procedimental, em outras palavras, o conhecimento implícito. Com isso, resulta na formação de representações mentais que serão armazenadas na memória de longo prazo.

Em relação aos fenômenos de variação e mudança sonora, duas categorias de frequência apresentam influência direta: a frequência de ocorrência (token) e a frequência de tipo. A primeira se refere à ocorrência de um dado item lexical em um corpus, ou seja, uma palavra que se repete em um texto, enquanto a última se refere a um padrão fonotático particular que se repete em palavras (BYBEE, 2002), como o passado regular do inglês, terminado em $-E D$.

De forma semelhante à visão de língua enquanto $\mathrm{SAC}$ e à $\mathrm{FU}$, o Modelo de Exemplares (ME) explica os efeitos de mudança através da exposição da língua mediante as experiências vividas pelo aprendiz. Segundo Bod e Cochran (2007, apud PIERREHUMBERT, 2006, p. 139) "Modelos de Exemplares objetivam capturar detalhadamente eventos linguísticos que falantes retêm, armazenando-os em exemplares ao longo do tempo e comparando-os a novas formas de input." Em outros termos, a representação cognitiva de uma palavra é constituída por um conjunto de exemplares de palavras utilizadas pelos falantes. Esses exemplares são categorizados em rede de associações 
entre palavras que, por sua vez, mapeiam relações de similaridade em todos os níveis fonético-fonológico, morfológico, sintático, semântico e pragmático (GOLDINGER, 1996).

Pierrehumbert (2006) advoga que ao perceber certa palavra, o falante armazena na memória informações fonéticas detalhadas assim como informações não linguísticas relacionadas ao input. $\mathrm{O}$ falante, ao se deparar com outra palavra a categoriza e armazena de acordo com a similaridade dos exemplares já presentes na memória.

Adicionalmente, Pierrehumbert (2016) ainda explica que uma categoria (por exemplo, um fonema) é representada por uma nuvem de exemplares, composta por todas as ocorrências ou exemplares guardados na memória. Exemplares semelhantes estão armazenados numa mesma categoria. A similaridade das características acústicas do exemplar é fator de ativação para o reconhecimento e nível das categorias de nuvem. Assim, o detalhe fonético do inglês-L1 seria adquirido de forma gradual, associado diretamente à maior ou menor recorrência dos padrões.

A breve explanação de algumas características dos modelos fonológicos multirepresentacionais, a FU e o ME, evidencia o seu distanciamento com relação a visões fonológicas associadas à aplicação de regras, processos, restrições e/ou ranqueamentos aplicados na percepção/produção da estrutura fonológica subjacente. O Quadro 1 busca apresentar de modo sucinto as diferenças entre a proposta de análise fonológica tradicional e a proposta dos modelos multirepresencionais.

Quadro 1 - Principais características dos modelos fonológicos tradicionais e multirepresentacionais.

\begin{tabular}{|c|c|}
\hline Proposta Tradicional & Modelos multirepresentacionais \\
\hline Representação mental minimalista & Representação mental detalhada \\
\hline Separação entre fonética e fonologia & Inter-relação entre fonética e fonologia \\
\hline $\begin{array}{c}\text { Visão da fonologia como uma gramática } \\
\text { formal, com a utilização de variáveis } \\
\text { abstratas }\end{array}$ & $\begin{array}{l}\text { Consideração de que a fonologia da } \\
\text { língua envolve a distribuição } \\
\text { probabilística de variáveis }\end{array}$ \\
\hline $\begin{array}{l}\text { Efeitos da frequência refletidos na } \\
\text { produção em curso e não armazenados } \\
\text { da memória de longo termo }\end{array}$ & $\begin{array}{c}\text { Efeitos da frequência armazenados na } \\
\text { memória de longo termo }\end{array}$ \\
\hline $\begin{array}{l}\text { Julgamento fonotático categórico: uma } \\
\text { sequência ou é considerada bem } \\
\text { formada ou é impossível de ocorrer na } \\
\text { língua }\end{array}$ & $\begin{array}{l}\text { Efeitos gradientes nos julgamentos } \\
\text { fonotáticos }\end{array}$ \\
\hline Léxico separado da gramática fonotática & Palavra como lócus da categorização \\
\hline
\end{tabular}

Fonte: Guimarães (2004, p. 40).

O paradigma de língua(gem) enquanto SAC vai de encontro ao modelo linguístico tradicional, determinístico, processual, afeito a regras. A aprendizagem de uma língua ocorre de modo não-linear, complexo e associado às condições iniciais de cada agente do sistema, indivíduos que buscam aprender o inglês-L2 (PAIVA, 2005, 2009). Por sua vez, a FU e o ME defendem a relevância da análise dos efeitos do uso linguístico, componente da estrutura fonológica por meio da incorporação do detalhe fonético à representação subjacente. Os referidos modelos teóricos vão ao encontro de um modelo linguístico focado na variação e na análise do detalhe fonético desta variação, harmonizando-se deste modo com os objetivos deste estudo. Passamos na próxima seção à discussão dos procedimentos metodológicos de realização da pesquisa.

\section{Metodologia}

Esta é uma pesquisa de corte transversal e desenho quase-experimental, como foco no detalhe fonético espectral e de duração dos pares vocálicos i:-I, $\mathfrak{a - \varepsilon}$ e u:-ひ do inglês-L2. As referidas vogais distinguem-se pelos diferentes pontos de realização no continuum acústico- 
articulatório e também pela duração, apresentando o primeiro componente de cada par maior duração que o segundo na fonologia do inglês-L1 (JONES, 1976).

O universo de pesquisa constituiu-se de alunas de cursos livres de inglês na cidade de Mossoró (RN). As informantes foram selecionadas tendo em vista seu nível de proficiência, sua inexperiência em países de língua inglesa, residência na região nordeste do país a maior parte da vida e pelo baixo contato com inglês fora da sala de aula.

Tivemos como variável dependente a realização acústica das vogais i:-I, $\mathfrak{a - \varepsilon}$ e u:- $\mathbf{0}$ do inglês-L2. Foram analisados dados em Hertz (Hz) dos primeiros dois formantes (F1 e F2), bem como da duração (em milissegundos - ms.) de cada vogal (FANT, 1960).

Foram selecionadas palavras contendo as vogais supracitadas entre oclusivas, para facilitar a medição da duração, em posição de meio de palavra em sílaba tônica inicial de dissílabas do PB, e.g. bica, teto, puta, e monossílaba do tipo fonotático CVC no inglês, e.g. deep, bit, pet, back, put, poop. Tomamos o cuidado de selecionar palavras cuja consoante seguinte à vogal analisada fosse uma desvozeada para homogeneizar a análise da duração. As referidas palavras foram primeiramente inseridas num experimento clássico de leitura de frases-veículo e posteriormente num mapa de uma pequena cidade, atividade próxima a um role-play e que buscou a coleta de dados num contexto com foco no uso por parte das informantes. Utilizamos metodologia de coleta e análise de dados acústicos recomendada por Ladefoged (2003), Johnson (2012), Strange, Schirru e MacKey (2003).

Além da palavra, outras variáveis independentes foram analisadas, como o nível de proficiência e experiência de uso no inglês-L2, frequência de ocorrência das palavras, bem como o comportamento de cada item lexical com relação à realização da vogal. Lembramos que neste artigo focamos a variável indivíduo, tentando enfatizar as implicações que a análise desta variável pode contribuir para o entendimento da aquisição do sistema vocálico do inglês-L2 por aprendizes brasileiros. Referências às outras variáveis que foram controladas serão feitas quando consideradas relevantes na análise e discussão dos dados.

As gravações foram realizadas em ambiente silencioso, uma vez que a utilização de salas com tratamento acústico não eram disponibilizadas pelos cursos livres. Utilizamos um gravador Zoom H6 e um microfone Shure SM58 para garantir a qualidade dos arquivos de áudio Wave não comprimidos. A análise acústica dos dados foi realizada com o programa PRAAT (BOERSMA; WEENINK, 2017), versão 6.0.37. Análises estatísticas foram performadas com o programa SPSS (IBM, 2011), versão 20.1 .

Neste artigo realizamos comentários e análises de cunho quali-quantitativo. Realizamos diversos testes estatísticos, a depender da natureza dos dados analisados. Utilizamos, por exemplo, a ANOVA de medidas repetidas e o Modelo Generalizado Linear (GLM), bem como utilizamos o teste $t$ para amostras pareadas. O nível de significância estatística foi estabelecido em $p<0,05$. A seleção e interpretação dos testes estatísticos utilizados seguiram preceitos apresentados por Dancey e Reidy (2019).

Com o intuito de enfatizarmos a relevância da análise do indivíduo nos dados na próxima seção, apresentamos primeiramente resultados agrupados de variação espectral e de duração, cuja análise seria suficiente em grande parte dos modelos fonológicos de análise vigentes. O posterior aprofundamento do entendimento dessas questões ocorre ao focarmos os mesmos dados no nível individual, demonstrando a relevância de análise do detalhe fonético e da variação dos agentes do sistema como proposto pelo paradigma de língua(gem) como SAC e pelos modelos fonológicos multirrepresentacionais.

\section{Análise e discussão dos dados}

Iniciamos a seção de análise e discussão dos dados com foco numa análise agrupada das realizações da distância euclidiana entre as vogais dos pares vocálicos i:-I, $\mathfrak{e}-\boldsymbol{\varepsilon}$ e u:-ひ do 
inglês-L2. Uma vez obtidos os valores em Hertz (Hz) de F1 e F2 das vogais supracitadas torna-se possível a determinação do ponto de realização vocálica num gradiente acústico. Uma vez que os dados de F1 referem-se ao eixo da altura vocálica (vertical), e os valores de F2 ao eixo anterior-posterior (horizontal) ao realizarmos a análise de dados de cada vogal, podemos então medir a distância de realização entre os pontos, em $\mathrm{Hz}$, determinando assim se as vogais de um determinado par são realizadas com maior ou menor distância entre seus componentes quando comparadas às vogais de outro par vocálico.

Assim, observamos na Figura $1^{22}$,

Figura 1 - Distância euclidiana entre os pares de vogais i:-I, $\mathfrak{X - \varepsilon}$ e u:- $\mathbf{U}$ do inglês-L2.

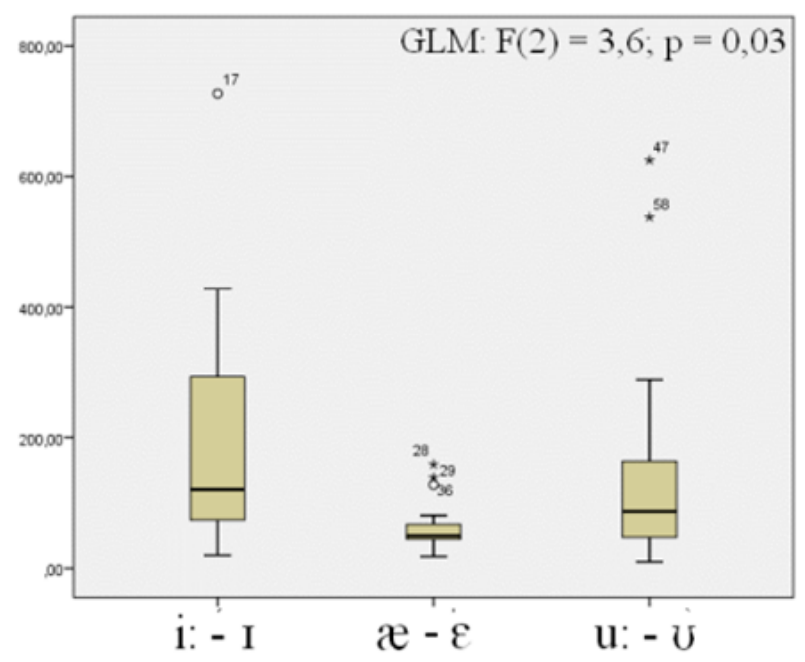

quanto maior a distância euclidiana (eixo vertical) envolvida na realização de um par vocálico, menor será a proximidade espectral entre seus pontos de realização. A análise gráfica descritiva indica pequena distância euclidiana no caso do par $\mathfrak{a}-\varepsilon$, o que significa dizer que ocorreu baixa distinção entre os pontos de realização das duas vogais do inglês-L2. Por sua vez, os pontos de realização do par anterior alto i:-I apresentam maior distância euclidiana entre as vogais do que a realização do par posterior alto u:-ひ. Os dados estatísticos inferenciais indicam a existência de diferença significativa entre as distâncias euclidianas dos referidos pares vocálicos, fato que nos permite apontar uma sequência decrescente de dificuldade de realização espectral das vogais com o par anterior baixo liderando, o par posterior alto numa posição intermediaria e, finalmente, o par anterior alto como o mais facilmente realizável no conjunto de dados: $\mathfrak{e}-\boldsymbol{\varepsilon}>\mathbf{u}:-\boldsymbol{\mho}>\mathbf{i}:-\mathbf{I}$.

Uma análise categórica de dados, como a anterior, seria suficiente para muitos modelos fonológicos tradicionais. Resultados como os anteriores vêm sendo consistentemente reportados pela literatura envolvendo a realização de vogais do inglês por aprendizes brasileiros. Todavia, defendemos que a análise do detalhe fonético de realização individual

Figura 2 - Plot vocálico das distâncias euclidianas entre as vogais i:-I do inglês-L2.

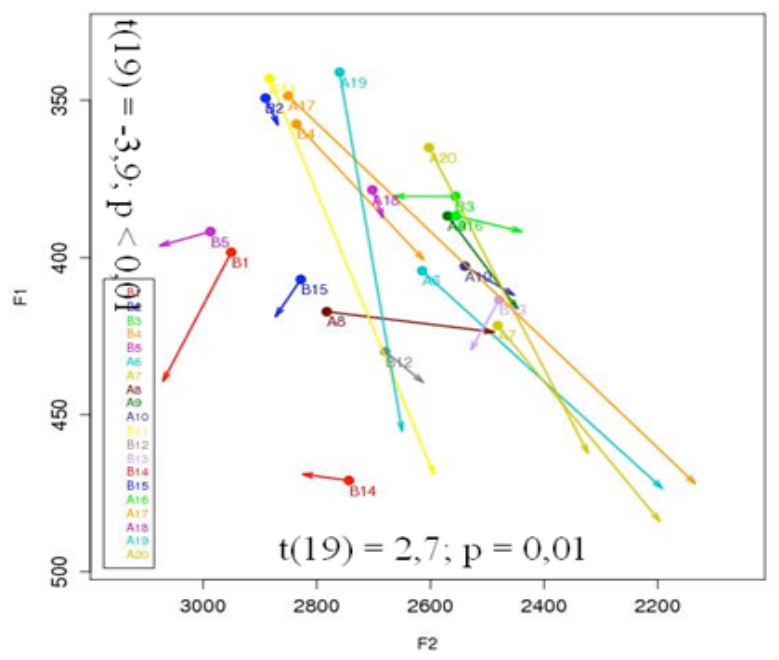
pode trazer valiosas informações à baila, ajudando na compreensão do percurso de construção da fonologia do inglês-L2 por aprendizes brasileiros. Portanto, passamos a seguir à reanálise do mesmo conjunto de dados espectrais, com foco no indivíduo conforme discutido anteriormente.

A Figura "2" apresenta dados individuais de realização da distância euclidiana entre as vogais do par anterior alto i:-I do inglês-L2. As letras iniciais indicam os alunos de nível advanced (avançado) e B para alunos nível Beginner (Iniciante). Em relação à numeração de 1 à 10 são alunas de um curso de idiomas e de 11 à 20 alunas de outro curso, com metodologias diferentes. Diferentemente da

\footnotetext{
${ }^{22}$ Todas as figuras são de autoria própria.
} 
Figura 1, os dados de F1 e F2 são apresentados em seus respectivos eixos vertical e horizontal, possibilitando assim a observação da distância euclidiana entre os pontos acústicos de realização das vogais. Adicionalmente, a Figura 2 torna possível a observação da direção da distância euclidiana entre os pontos vocálicos analisados. A vogal i: é representada no gráfico pelo ponto inicial, enquanto a vogal I é representada pela seta ao final de cada linha.

A comparação entre os pontos de realização acústica das vogais i:-I do inglês-L2 indicou diferença estatística significativa tanto no eixo F1 (de variação vertical) quanto no F2 (de variação horizontal) entre as vogais. Os resultados corroboram os dados anteriores, que indicaram maior distância euclidiana entre as vogais deste par.

A riqueza de detalhe fonético gradiente observado na Figura 2, todavia, apresenta mais informação sobre os distintos percursos individuais de construção da gramática fonológica do inglês. Algumas informantes apresentam grande distância euclidiana entre as vogais e outras apresentam quase diferença alguma. As informantes A19, A17 e B11 se destacam por apresentarem comportamento como esperado, recuo e abaixamento do ponto de realização quando comparado ao ponto de realização da vogal i: com I. Outras apresentam pouca variação, como A18, A10, A9 e B2. As informantes B1, B3, B14, B15 e B5 realizam movimento contrário comparando o ponto de realização da vogal $\mathbf{i}$ : ao ponto da vogal $\mathbf{I}$, cujo recuo esperado seria para o lado direito, com a segunda vogal em posição mais posterior, sugerindo a emergência de uma nova categoria vocálica distinta no inglês-L2. A análise do detalhe fonético individual de realização das distâncias euclidianas das vogais i:-I na Figura 2, dessa forma, apresenta a construção da gramática fonológica de forma mais complexa do que a análise na comparação entre os pares vocálicos realizados na Figura 1.

Fato semelhante é observado ao analisarmos os dados apresentados na Figura 3, envolvendo a distância euclidiana entre os pontos de realização do par vocálico anterior baixo $\mathfrak{x}-\varepsilon$ do inglês-L2. Tendo em vista as características espectrais do par, a vogal $\boldsymbol{\varepsilon}$ é representada no gráfico pelo ponto inicial, enquanto a vogal $\mathfrak{x}$ é representada pela seta ao final de cada linha. Lembramos que o referido par vocálico apresentou a menor distância euclidiana entre os três pares analisados, indicando ser o par cujas vogais são mais difíceis de serem separadas no espectro vocálico neste artigo. A observação da Figura "3" revela, de modo geral, pequenas distâncias euclidianas realizadas pelas informantes. Concomitantemente, os pontos de realização variam de forma mais marcante no eixo F2 (horizontal) do que F1 (vertical). O fato é contraintuitivo, uma vez que o par vocálico $\mathfrak{x}-\boldsymbol{\varepsilon}$ do inglês caracteriza-se na maioria de seus dialetos por uma variação no eixo vertical (F1) e não no horizontal (F2) (PETERSON; BARNEY, 1952; JONES, 1976). A análise estatística dos dados indicou apenas diferença não significativa no eixo $\mathrm{F} 1$, mas diferença significativa no eixo F2.

O resultado poderia apontar uma tendência das informantes deste estudo por uma auto-organização semelhante à encontrada no Northern Cities Vowel Shift, fenômeno linguístico americano do norte em que as vogais $\mathfrak{e}-\boldsymbol{\varepsilon}$ passam por um

Figura 3 - Plot vocálico das distâncias euclidianas entre as vogais $\boldsymbol{\varepsilon}$-æ do inglês-L2.

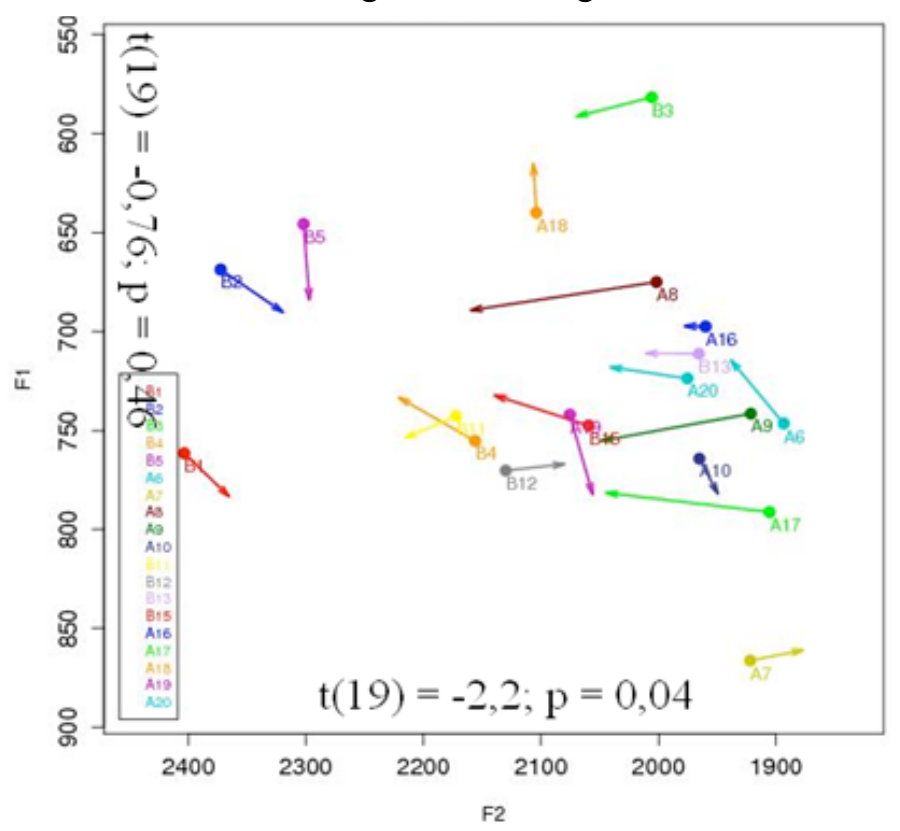


fenômeno de fusão, em que as duas vogais aproximam-se e tendem a amalgamar-se do ponto de vista espectral, passando a serem diferenciadas pela duração (LABOV; ASH; BOBERG, 2006). Todavia, a análise individual na Figura 3 indica a inexistência de um padrão específico, encontrado no inglês de cidades como Chicago e Detroit, que aponta uma centralização da vogal $\varepsilon$ e um alteamento e anteriorização da vogal $\mathfrak{x}$ (LABOV; ASH; BOBERG, 2006). A análise estatística dos dados espectrais das vogais $\mathfrak{x}-\boldsymbol{\varepsilon}$ do inglês-L2 com a vogal $\varepsilon$ do $\mathrm{PB}$ indica que as vogais do inglês-L2 são significativamente mais baixas $(\mathrm{F}(2)=$ $15,9 ; p<0,01)$ e centrais $(\mathrm{F}(2)=22,2 ; p<0,01)$ que a vogal do PB. A constatação indica uma auto-organização inesperada do sistema, uma vez a literatura reporta dificuldade na utilização do espaço acústico-articulatório anterior baixo na realização da vogal $\mathfrak{x}$ do inglês por aprendizes brasileiros (BARBOZA, 2008; LIMA JR., 2012). Enfatizamos que dados apresentados posteriormente neste estudo envolvendo a realização da duração dos pares de vogais indicará que o par vocálico anterior baixo $\mathfrak{e}-\varepsilon$ do inglês-L2 também apresenta maior grau de dificuldade quando comparado com os outros pares vocálicos desta pesquisa.

Em se tratando da análise individual qualitativa, observamos casos de variação semelhante ao inglês norte-americano associado ao Northern cities vowel shift, como nas informantes A8, A9 e A17. Todavia o fato é que cada indivíduo comporta-se de maneira distinta na autoorganização de seu espaço vocálico anterior baixo do inglês, com pequena distinção entre as vogais conforme indicado pelas distâncias euclidianas: A9 realiza o eixo da altura mais estável, porém ela realiza ambas as vogais em posição mais posterior do que o esperado. B1, B2, B5 e A19 realizam pequena distância euclidiana entre as vogais $\mathfrak{x}-\varepsilon$ do inglês-L2, já A16 realiza distância euclidiana muito pequena entre o ponto de realização de cada vogal, conforme podemos observar na Figura 3. Apesar de a análise estatística reportar diferença significativa no eixo F2 (horizontal), a conclusão é pela inexistência de distinção espectral significativa entre as vogais anteriores baixas $\mathfrak{x}-\boldsymbol{\varepsilon}$ do inglês-L2, à exceção de informantes isolados, fato que reforça a percepção que necessária se faz a análise individual dos dados tendo em vista que a auto-organização do sistema fonológico tem o potencial de revelar padrões inesperados de aquisição fonológica.

Passamos neste momento à discussão dos dados relativos à distância euclidiana individual na realização do par posterior alto u:- $\mathbf{U}$ do inglês-L2, apresentada na Figura 4. A vogal u: é representada no gráfico pelo ponto inicial, enquanto a vogal $\boldsymbol{U}$ é

Figura 4 - Plot vocálico das distâncias euclidianas entre as vogais $u:-\mho d o$ inglês-L2.

representada pela seta ao final de cada linha.

Observamos na Figura "4"
indica uma tendência pela
diferenciação entre as vogais posteriores altas u:- $\boldsymbol{\sigma}$ do inglês-L2 no eixo F2 (horizontal), com baixa variação no eixo F1 (vertical). A percepção impressionista dos dados individuais de distância euclidiana entre as vogais é corroborada pelos testes estatísticos, com diferença significativa sendo encontrada no eixo F2, mas apenas diferença não significativa reportada no eixo $\mathrm{F} 1$.

É relevante retomar que os dados de todas as informantes amalgamados na Figura 1 indicou um

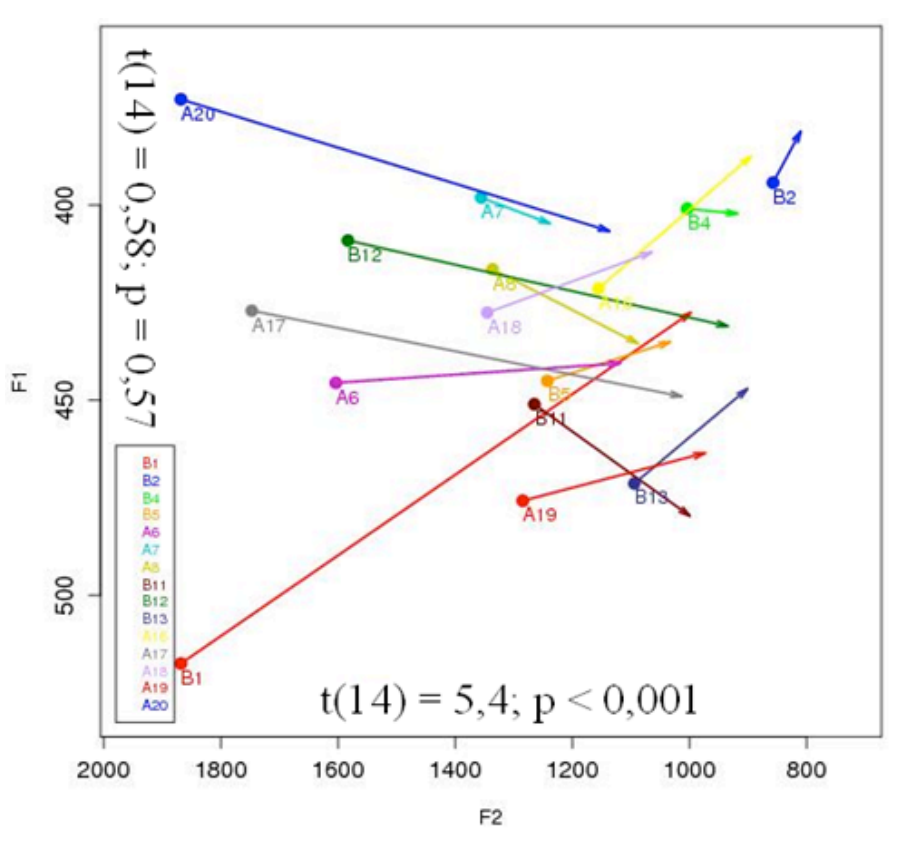


padrão de distância euclidiana intermediário do par vocálico posterior alto u:- $\mathbf{U}$ do inglês-L2 quando comparados aos pares i:-I e $\mathfrak{a}-\boldsymbol{\varepsilon}$. A interpretação visual coaduna-se com os resultados anteriores, uma vez que a realização do par anterior alto i:-I apresentou diferença significativa nos dois eixos (Figura 2) e o par anterior baixo $\mathfrak{x}-\varepsilon$ mal atinge a significância estatística no eixo horizontal (F2), apresentando baixos valores de distância euclidiana associados à maioria dos indivíduos (Figura 3).

Em se tratando da variação individual analisada de forma qualitativa, são observáveis grandes distâncias euclidianas diferenciando os pontos de realização das vogais u:-ひ no espaço vocálico. As informantes A20, B12, A17 e A6 apresentam marcantes distâncias euclidianas, com a vogal u:, que segundo a literatura deveria ser realizada num ponto mais posterior e alto, sendo de fato realizada de modo mais central em comparação à vogal $\boldsymbol{\sigma}$. É relevante enfatizar que a maior distância euclidiana entre as vogais posteriores é realizada pela informante B1. O fato indica que a referida informante construiu seu espaço vocálico posterior alto de modo inesperado, mas com dois pontos de realização vocálicos marcadamente distintos um do outro. Ao observamos os dados individuais de B1 no caso dos pares vocálicos discutidos anteriormente, concluímos que naqueles casos específicos a informante não apresenta variação individual marcante entre as vogais dos pares i:-I e $\mathfrak{e}-\boldsymbol{\varepsilon}$. Pode-se hipotetizar portanto percursos de construção da interfonologia PB/inglês-L2 também em nível fonêmico. Adicionalmente, temos informantes com variação intermediária na distância euclidiana entre as vogais posteriores u:- $\boldsymbol{\mho}$ do inglês-L2, como A18, A19 e B11, ou apenas pequenas distâncias, como B4 e B2.

A análise individual de realização da distância euclidiana entre as vogais posteriores $\mathbf{u}:-\boldsymbol{\sigma}$ evidencia que todas as informantes realizaram a vogal $\mathbf{u}$ : num ponto mais central do que a vogal $\boldsymbol{\sigma}$. Na literatura encontramos estudos que podem ajudar na melhor compreensão do conjunto de dados, bem como da marcante variação individual reportada anteriormente. $\mathrm{O}$ fenômeno de GOOSE-fronting envolve a anteriorização da vogal u: reportada em diversos países cujo idioma nativo é o inglês (BARANOWSKI, 2017). O fenômeno de anteriorização da vogal posterior alta já vem sendo reportado na fala de imigrantes (WONG, 2014), e mesmo de aprendizes brasileiros de inglês-L2 (MARUSSO, 2002). A observação do detalhe fonético associado às vogais posteriores, bem como sua análise qualitativa individual quanto à distância e direção da variação, permite hipotetizar que o sistema fonológico do inglês nativo, em constante mudança dinâmica, devido à sua abertura para variáveis externas e portanto em constante auto-organização, apresenta o potencial de influenciar a realização do espaço

Figura 5 - Diferença de duração entre os pares de

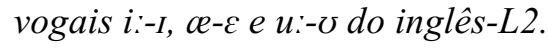

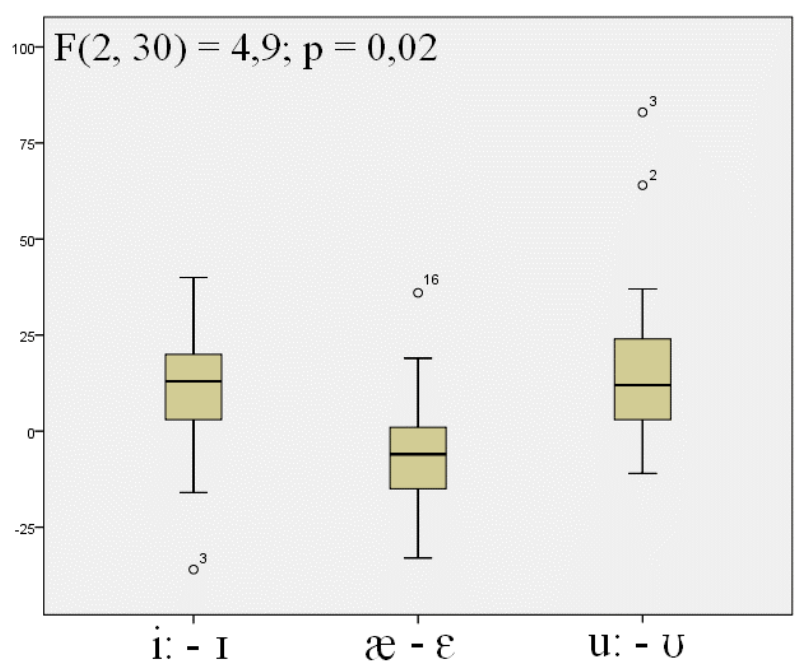
vocálico de aprendizes brasileiros, conforme inferência autorizada pelos dados apresentados na Figura 4. Naturalmente, a hipótese de uma mudança em progresso marcadamente associada ao inglês-L1, como o GOOSE-fronting, exercer influência significativa na Aquisição de inglês-L1 por aprendizes brasileiros(as) deve ser aprofundada por estudos adicionais. $\mathrm{O}$ fato, todavia, não impede a conclusão que as línguas envolvidas na interfonologia espectral ora discutida são SACs, cuja interação ocorre ao mesmo tempo entre e dentre sistemas, além do nível individual, conforme observado nos dados analisados.

Uma vez realizadas diversas considerações acerca da variação espectral individual 
envolvendo os pares vocálicos analisados nesta pesquisa numa perspectiva complexa e com foco no detalhe fonético, passamos a partir deste ponto à análise dos dados de duração vocálica.

Conforme metodologia empregada na discussão anterior, apresentamos primeiramente os dados de forma amalgamada, com foco na diferença de duração entre as vogais dos pares $\mathbf{i}:-\mathbf{I}, \mathfrak{x}-\boldsymbol{\varepsilon}, \mathbf{u}:-\boldsymbol{\mho}$ do inglês-L2 na Figura "5". A diferença entre as durações foi calculada subtraindo a duração da primeira vogal de cada par pela duração da segunda. É esperado que a diferença de duração entre as vogais seja positiva, uma vez que a primeira vogal de cada par apresenta maior duração que a segunda na fonologia do inglês (JONES, 1976). O que emerge da análise amalgamada dos dados de duração neste caso é que os pares vocálicos i:-I e u:-ひ apresentam nível semelhante de realização da duração, mais uma vez com o par $\mathfrak{x - \varepsilon}$ apresentando dificuldade para os informantes. A análise qualitativa é corroborada pela diferença estatística significativa entre os dados dos pares vocálicos. De forma semelhante ao reportado na análise do detalhe fonético espectral, a análise amalgamada dos dados envolvendo duração dos pares vocálicos é relativamente pouco esclarecedora quando comparada à análise de dados individuais de diferença de duração entre os pares apresentada na Figura " 6 ".

Figura 6 - Diferença de duração entre os pares de vogais i:-I, $\mathfrak{e - \varepsilon}$ e u:-ひ do inglês-L2 por informante em ordem crescente.

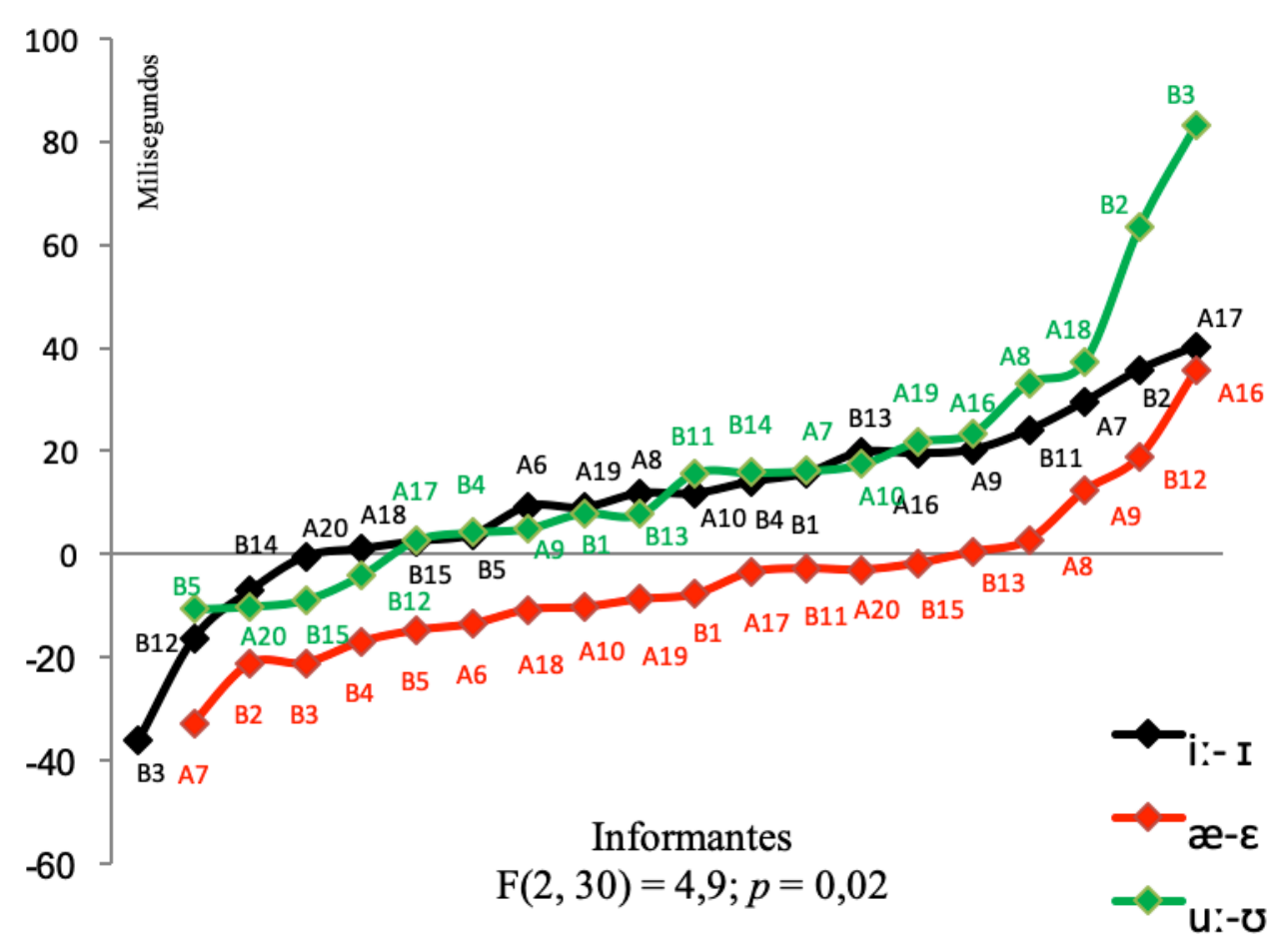

Os dados na Figura 6 apontam, de modo geral, pequena diferença na duração entre as vogais dos pares i:-I, $\mathfrak{x - \varepsilon}, \mathbf{u}:-\boldsymbol{\sigma}$ do inglês-L2, sendo esse um indicativo que informantes usam a duração de modo distinto do padrão fonológico do inglês-L1. Um elevado número de informantes realizou diferença negativa de duração. Isso implica dizer que a vogal curta foi realizada com maior duração que a longa nesses casos. A constatação é indicada por todas as informantes que estão abaixo da linha horizontal e central na figura, associada ao valor 0 (zero) milissegundos de diferença de duração entre as vogais. A linha vermelha que representa as diferenças de duração do par anterior baixo apresenta maior número de 
ocorrências negativas, indicando ser o par mais problemático para as informantes. Por sua vez valores relativos aos pares vocálicos anterior alto e posterior alto são bastante aproximados, à exceção dos valores extremos em ambos os casos. A análise estatística dos dados indica a existência de diferença significativa envolvendo a realização da diferença de duração entre os pares vocálicos i:-I, $\mathfrak{x}-\boldsymbol{\varepsilon}, \mathbf{u}:-\boldsymbol{\mho}$ do inglês-L2. Conclui-se que, de modo semelhante ao que ocorreu com relação ao espectro acústico, o par anterior baixo $\mathfrak{x}-\varepsilon$ também representa desafio considerável de aquisição do detalhe fonético de duração das vogais envolvidas.

A análise individual dos dados aponta percursos marcadamente distintos de realização da duração nos pares vocálicos analisados. Consideremos por exemplo a informante A16, cuja diferença na duração entre as vogais $\mathfrak{a}-\boldsymbol{\varepsilon}$ do inglês-L2 teria o potencial de caracterizá-la como uma boa aprendiz desse detalhe fonético específico envolvendo a realização vocálica, uma vez que neste par encontramos o maior desafio para aprendizes brasileiros segundo os dados deste estudo. De fato, a informante A16 encontra-se entre as que apresentam maior diferença de duração envolvendo os dados dos pares i:-I e u:-o do inglês-L2, sem, todavia, demonstrar a mesma facilidade de controle da emergência do detalhe fonético espectral na organização de seu sistema fonológico vocálico do inglês-L2.

Por sua vez, a informante B3 apresenta a maior duração positiva na realização da duração do par posterior alto $\mathbf{u}:-\boldsymbol{\mho}$, indicando que ela adquiriu as duas categorias de duração no caso do par vocálico posterior alto. Entretanto, quando esperamos que esta habilidade de B3 em adquirir a duração emergisse naturalmente nos outros pares, por meio de uma autoorganização de todo o sistema, observamos que não é o que acontece: no caso do par anterior alto i:-I, B3 apresenta a maior diferença negativa, indicando que realizou a diferença de duração neste par de modo totalmente inverso ao esperado. Fato semelhante ocorre no caso do par anterior baixo $\mathfrak{a}-\boldsymbol{\varepsilon}$, em que B3 apresentou a terceira realização mais distante do esperado no conjunto de dados.

Ao confrontarmos tais resultados com as informações encontradas em seus respectivos questionários de anamnese, identificamos comportamentos individuais de auto-organização do sistema vocálico de difícil predição. Retomando o caso da informante B3, raramente a referida informante estuda pronúncia e pratica conversações em inglês, seja com outros aprendizes de inglês-L2 ou com falantes nativos. Seria esperado então que B3 tivesse dificuldade na realização da duração dos três pares de vogais, algo que ocorre apenas em dois casos, como discutido anteriormente. A informante A7 estuda pronúncia na maioria das vezes, além de praticar conversação em inglês com falantes nativos. Seria esperado então que A7 não apresentasse dificuldade na realização da duração dos três pares de vogais. Conforme esperado, A7 apresenta maior diferença de duração entre as vogais nos pares anterior alto i:-I e anterior baixo $\mathfrak{a}-\boldsymbol{\varepsilon}$, mas não no par posterior alto u:- $\mathbf{\sigma}$. Como explicar então a emergência da maior diferença de duração entre as vogais na Figura "6" favorável a B3?

$\mathrm{O}$ indivíduo enquanto SAC comporta-se de modo não-linear, com marcante variação e constrói sua interfonologia $\mathrm{PB} /$ inglês-L2 de modo emergente e à borda do caos. Segundo Larsen-Freeman (2008) tendo em vista que cada sujeito é um sistema complexo, todos os componentes estão interligados entre eles. Sendo assim, diversas variáveis podem influenciar a informante, acarretando que ela adquira a relação de duração do par posterior alto por alguma variável de difícil, ou mesmo impossível, determinação. Pode-se hipotetizar por exemplo que B3 incorporou à sua gramática fonológica em construção no inglês-L2 a diferença de duração entre as vogais u:-ひ nos pares mínimos de palavras como food-foot, pool-pull e Luke-look. Todavia, o evento catalizador desta incorporação, a pedra causadora da avalanche, qualquer que seja, foi aparentemente de baixo impacto, sendo incapaz de alterar o sistema atrator associado à realização não-padrão encontrada no caso dos pares anterior alto i:-I e anterior baixo $\mathfrak{x}-\boldsymbol{\varepsilon}$. Encerramos neste momento a seção de análise e discussão de dados do estudo. Apresentamos a seguir as considerações finais da pesquisa. 


\section{Considerações finais}

Esta pesquisa teve como objetivo geral descrever a emergência da interfonologia vocálica do português brasileiro (PB) e do inglês-L2 na realização de aprendizes brasileiros a partir de uma perspectiva linguística complexa, multirrepresentacional e focada no indivíduo.

A característica emergente da auto-organização individual da construção da gramática fonológica envolvendo o sistema vocálico do inglês-L2 é observável caso o pesquisador busque instrumentos de análise que propiciem o foco na variação e no detalhe fonético, caso desta pesquisa. Dados os resultados envolvendo a variação individual e o detalhe fonético de realização dos pares de vogais i:-I, $\mathfrak{x - \varepsilon , ~ u : - \mho}$ do inglês-L2 apresentados na seção anterior, tomamos a hipótese básica da pesquisa, que envolve a auto-organização individual no percurso de construção da gramática fonológica do inglês-L2, como confirmada.

A análise poderia ser mais aprofundada a partir do cruzamento dos dados relativos às informações de detalhe fonético espectrais e de duração por indivíduo. Adicionalmente, o modelo de coleta de dados com corte transversal apresenta apenas uma fotografia do sistema fonológico das informantes. Do ponto de vista da língua(gem) enquanto SAC, seria mais pertinente a análise de dados longitudinais. Estas limitações constituem-se desdobramentos lógicos deste estudo, a serem realizados posteriormente.

Em resumo, esperamos ter apresentado os benefícios de análise dados da interfonologia PB/inglês-L2 de numa perspectiva complexa, multirrepresentacional e como foco no indivíduo e no detalhe fonético. Tal metodologia tem o potencial de aprofundar significativamente $\mathrm{o}$ entendimento da construção deste conhecimento por aprendizes brasileiros de inglês-L2.

\section{Referências}

BACCHI, Annalisa. Construction learning as a complex adaptive system: psycholinguistic evidence from L2 learners of English. Heidelberg: Springer International Publishing, 2015. BAPTISTA, Barbara O. The acquisition of English vowels by Brazilian-Portuguese speakers. Florianópolis: UFSC, 2000.

BARBOZA, Clerton Luiz Felix. Descrição acústica dos sons vocálicos anteriores do inglês e do português realizados por professores de inglês língua estrangeira no oeste potiguar. 182f. Dissertação (Mestrado em Linguística Aplicada) - Programa de Pós-Graduação em Linguística Aplicada, UECE, 2008.

BARANOWSKI, Maciej. Class matters: the sociolinguistics of GOOSE and GOAT In: Manchester English. Language Variation and Change, n. 29, p. 301-339, 2017.

BECKNER, Clay et al. Language is a complex adaptive system: position paper. Language Learning, v. 59, sup. 1, p.1-26, 2009.

BION, Ricardo A. et al. Category formation and the role of spectral quality in the perception and production of English front vowels. In: INTERSPEECH, 9a ed., 2006. Proceedings of Interspeech. Pittsburgh: ISCA. p. 1363-1366.

BOERSMA, Paul, WEENINK, David. Praat: doing phonetics by computer. Versão 6.0.37. Disponível em: <http://www.praat.org>. 2017.

BYBEE, Joan L. The phonology of the lexicon: Evidence from lexical diffusion. In: BARLOW, Michael; KEMMER, Suzanne (eds.). Usage-based models of language. Stanford: CSLI, 2000, p. 65-85.

Phonology and language use. Cambridge: Cambridge University Press, 2001.

Phonological evidence for exemplar storage of multiword sequences. Studies in

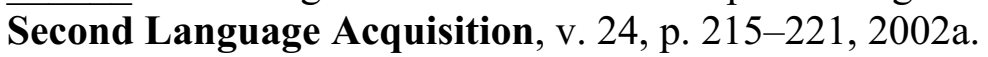

Word frequency and context of use in the lexical diffusion of phonetically

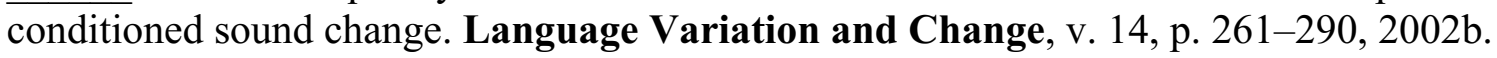


. Usage-based grammar and second language acquisition. In; ROBINSON, P.;

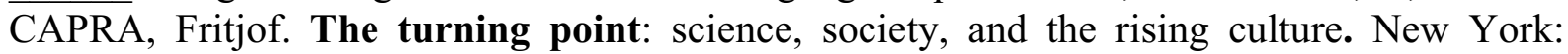
Bantam, 1983.

CILLIERS, Paul. Complexity and postmodernism: understanding complex systems. London: Routledge, 1998.

COOPER, David L. Linguistic attractors: The cognitive dynamics of language acquisition and change. Amsterdam: John Benjamins, 1999.

DANCEY, Christine P.; REIDY, John. Estatística sem matemática para psicologia: usando SPSS para Windows. $7^{\text {a }}$ Edição. Porto Alegre: Artmed, 2019.

DE BOT, Kees. Introduction: second language development as a dynamic process. The Modern Language Journal, v. 92, n. 2, p. 166-179, 2008.

; LOWIE, Wander; VERSPOOR, Marjolijn. A Dynamic Systems Theory approach to second language acquisition. Bilingualism: Language and Cognition, v. 10, n. 1, p. 7-21, 2007. ELLIS, Nick. Handbook of cognitive linguistics and second language acquisition. New York: Routledge, 2008.

. Language, usage and cognition. Cambridge: Cambridge University Press, 2010.

; BECKNER, Clay. Usage Based Theory. In: HEINE, Bernd; NARROG, Heiko. The

Oxford Handbook of Linguistics Analysis. Cambridge University Press, 2010, p. 827-855.

FANT, Gunnar. Acoustic theory of speech production: with calculations based on x-ray studies of Russian articulators. The Hague: Mouton, 1960.

FLEGE, James Emil. Second language speech learning: theory, findings, and problems. In:

GLEICK, James. Chaos: making a new science. New York: Viking, 1987.

GOLDINGER, Stephen D. Words and voices: episodic traces in spoken word identification and recognition memory. Journal of Experimental Psychology: learning, memory, and cognition, v. 22, n. 5, p. 1166-1183, 1996.

GUIMARÃES, Daniela Maria Lima Oliveira. Sequências de (sibilante+africada alveolopalatal) no português falado em Belo Horizonte. 122 f. Dissertação (Mestrado em Linguística) - UFMG, Belo Horizonte, 2004.

HOLLAND, John. Hidden Order: How Adaptation Builds Complexity. Cambridge: Perseus Books, 1995.

IBM. Statistical Package for the Social Sciences. Versão 20.1. Armonk, NY: IBM Corp, 2011.

JOHNSON, Keith. Acoustic and auditory phonetics. 3 ed. Malden, Ma: Blackwell, 2012.

JONES, Daniel. An outline of English phonetics. $9^{\text {th }}$. Ed. Cambridge: Cambridge University Press, 1976.

KRAMSCH, Claire. Why is everyone so excited about complexity theory in applied linguistics? Mélanges Crapel, n. 33, p. 9-24, 2012.

LABOV, William; ASH, Sharon; BOBERG, Charles. The atlas of North American English: phonetics, phonology and sound change. Berlin: Mouton de Gruyter, 2006.

LADEFOGED, Peter. Phonetic data analysis: an introduction to fieldwork and instrumental techniques. Malden: Blackwell Publishing, 2003.

LARSEN-FREEMAN, Dianne. Chaos, Complexity Science and Second Language Acquisition. Applied Linguistics. v. 18, n. 2, p. 141-165, 1997.

; CAMERON, Lynne. Complex systems in first and second language development. In: LARSEN-FREEMAN, Dianne; CAMERON, Lynne. Complex systems and applied linguistics. Oxford: OUP, 2008. p. 115-160

LEWIN, Roger. Complexity: life at the edge of chaos. New York: Macmillam Publishing Company, 1992.

LIMA JR, Ronaldo, M. A influência da idade na aquisição da fonologia do inglês como língua estrangeira por brasileiros. 187f. Tese (Doutorado em Linguística) - UNB, Brasília, 2012. 
LORENZ, Edward N. Deterministic nonperiodic flow. Journal of the Atmospheric Sciences, n. 20, p.130-141, 1963.

MARUSSO, Adriana S. Redução vocálica: estudo de caso no português brasileiro e inglês britânico. 513f. Tese (Doutorado em Linguística) - UFMG, Belo Horizonte, 2003.

PAIVA, Vera L. M. O. Modelo fractal de aquisição de línguas. In: BRUNO, F. C. (Org.) Reflexão e Prática em ensino/aprendizagem de língua estrangeira. São Paulo: Editora Clara Luz, 2005. p. 23-36

. Caos, complexidade e aquisição de segunda língua. In: PAIVA, Vera Lúcia Menezes de Oliveira; NASCIMENTO, Milton do. Sistemas adaptativos complexos: lingua(gem) e aprendizagem. Belo Horizonte: Letras/UFMG, 2009. p. 187-203.

PETERSON, Gordon E.; BARNEY, Harold L. Control Methods used in a study of the vowels. Journal of the Acoustical Society of America, v. 24, n. 2, p. 175-184, 1952.

PIERREHUMBERT, Janet B. What people know about sounds of language. Studies in the Linguistic Sciences. v. 29, n. 2, 1999. p. 111-120.

Exemplar dynamics: Word frequency, lenition, and contrast. In: BYBEE, Joan.; HOPPER, Paul.(eds.). Frequency effects and the emergence of lexical structure. John Benjamins, Amsterdam. 2001, p.137-157.

2002. p. 101-139.

Word-specific phonetics. Laboratory Phonology VII. Berlin: Mouton de Gruyter,

. The next toolkit. Journal of Phonetics. v. 34, n. 4, p. 516-530, 2006.

Phonological representation: Beyond abstract versus episodic. Annual Review of Linguistics 2, 2016, p. 33-52.

RAMIRES, Helena R. Percepção e produção de vogais coronais do inglês por falantes nativos de português brasileiro: o papel da instrução explícita. 78f. Dissertação (Mestrado em Letras) - Universidade Católica de Pelotas, 2016.

RÁUBER, Andréia S. Perception and production of English vowels by Brazilian EFL speakers. 203f. Tese (Doutorado em Letras) - Universidade Federal de Santa Catarina, Florianópolis, 2006.

STRANGE, Winifred. Speech perception and linguistics: theoretical and methodological issues. Tinomium: York Press, 1995.

. Age of learning and second language speech. In: BIRDSONG, David. (Org.) Second language acquisition and the Critical Period Hypothesis. Mahwah: Lawrence Erlbaum Associates, 1999. p. 101-131.

; SCHIRRU, Carlo; MACKAY, Ian R. A.; Interaction between the native and second language phonetic subsystem. Speech communication, v. 40, p. 467-491, 2003.

VAN GEERT, Paul.; VAN DIJK, Marijin. Focus on variability: new tools to study intraindividual variability in developmental data. Infant Behavior \& Development, v. 25, p. 340374, 2002.

WALDROP, M. Mitchell. Complexity: the emerging science at the edge of order and chaos. New York: Touchstone, 1993.

WONG, Amy Wing-mei. GOOSE-fronting among Chinese Americans in New York City. University of Pennsylvania Working Papers in Linguistics. v. 20, n. 2. 2014. p. 209-218.

ZIMMER, Márcia C. A transferência do conhecimento fonético-fonológico do português brasileiro (L1) para o inglês (L2) na recodificação leitora: uma abordagem conexionista. 187 f. Tese (Doutorado em Letras) - PUC-RS, Porto Alegre, 2003.

Recebido em 08/06/2019

Aceito em 24/08/2019 